\title{
Identificación de especies de Naegleria en sitios recreativos en Hornos, Sonora
}

\section{Identification of Naegleria species in recreational areas in Hornos, Sonora}

\author{
Eunice Guzmán-Fierros ${ }^{1}$, Johan F. De Jonckheere ${ }^{2}$ y Fernando Lares-Villa ${ }^{1 *}$ \\ ${ }^{1}$ Departamento de Ciencias Agronómicas y Veterinarias, Instituto Tecnológico de Sonora. 5 de Febrero 818 Sur, 85000, Cd. Obregón, Sonora, \\ México \\ ${ }^{2}$ Scientific Institute of Public Health, B-1050 Brussels, Belgium \\ *Correspondencia: flares@itson.mx
}

\begin{abstract}
Resumen. Por medio de técnicas moleculares se han identificado más de 47 especies del género Naegleria, pero en los estudios hechos en México sólo 4 han sido identificadas. El objetivo de este estudio fue identificar el mayor número de Naegleria spp. en sitios recreativos en Hornos, Sonora. Para ésto, se seleccionaron 9 sitios que se muestrearon durante los meses de junio a septiembre de 2004. Se identificaron genéticamente 15 especies aisladas mediante secuenciación de DNA ribosomal, 9 ejemplares como $N$. lovaniensis, 5 como $N$. tihangensis y 1 como $N$. americana. Es la primera vez que se registra la presencia de $N$. americana y $N$. tihangensis en la región; esta última especie está muy relacionada con otra amiba patógena, $N$. australiensis. Una de las cepas aisladas de $N$. lovaniensis resultó ser única porque difiere en 1 par de bases de las otras cepas de esta especie.
\end{abstract}

Palabras clave: amibas, ITS, 5.8S rDNA, México.

\begin{abstract}
By means of molecular techniques more than 47 species of the genus Naegleria have been identified, but in Mexico, only 4 of these species have been so identified. The objective of this study was to identify a higher number of Naegleria spp. in recreational areas in Hornos, Sonora. Nine sites were selected and sampling was performed from June to September of 2004. Fifteen isolated species were identified genetically by means of sequencing ribosomal DNA, 9 specimens as $N$. lovaniensis, 5 as $N$. tihangensis and 1 as $N$. americana. It is the first time that $N$. americana and $N$. tihangensis are reported in this region. The latter species is closely related to another pathogenic amoeba, $N$. australiensis. One of the isolated strains of $N$. lovaniensis is unique because it differs by $1 \mathrm{bp}$ from the other strains of this species.
\end{abstract}

Key words: amebas, ITS, 5.8S rDNA, Mexico.

\section{Introducción}

La especie Naegleria fowleri (Carter) es el agente causal de la meningoencefalitis amibiana primaria (MAP), infección aguda de evolución rápida y fatal en la mayoría de los casos, que se presenta en individuos jóvenes y saludables con historial reciente de natación u otras actividades en aguas cálidas (Vargas-Zepeda et al., 2005). Otras 2 especies, $N$. australiensis (De Jonckheere) y N. italica (De Jonckheere, Pernin, Scaglia y Michel), han mostrado ser patógenas en pruebas de laboratorio con animales y se consideran potencialmente patógenas para humanos. Durante estudios internacionales en la búsqueda de la amiba patógena, se encontró que había más de las 2 especies conocidas originalmente, $N$. gruberi (Schardinger) y $N$. fowleri. En la actualidad, se han identificado 47 especies (Cuadro 1) pertenecientes a este género, muchas de las cuales se

Recibido: 18 septiembre 2006; aceptado: 12 junio 2007 han identificado por primera vez, y otras reclasificado con base en sus secuencias genéticas (De Jonckheere et al., 2001; De Jonckheere, 2002; De Jonckheere, 2004; De Jonckheere y Brown, 2005; Visvesvara et al., 2005; De Jonckheere, 2006a, b). El objetivo de este trabajo fue aislar e identificar especies de Naegleria en diversos cuerpos de aguas naturales superficiales con uso recreativo, mediante los métodos convencionales y también por el uso de secuencias de rDNA.

\section{Materiales y métodos}

Se seleccionaron los siguientes medios acuáticos y sitios de interés recreativo en el área de Hornos, dentro de la región sur de Sonora: la presa Álvaro Obregón, la laguna en parque Oviachic, fuente termal de Aguacaliente, 3 canales, uno en cada uno de los paseos Las Palmas, La Isleta, y El Álamo, la represa Chiculi del ríoYaqui, el dique 
Cuadro 1. Especies de Naegleria descritas en la actualidad, temperatura máxima de crecimiento y características de flagelación

\begin{tabular}{|c|c|c|c|}
\hline Especies & Autor, año & $T^{\circ} \mathrm{C}$ & Flagelación \\
\hline N. gruberi & Schardinger, 1899, De Jonckheere, 2002 & 39 & + \\
\hline N. fowleri & Carter, 1970 & 45 & + \\
\hline N. jadini & Willaert y Le Ray, 1973 & 35 & + \\
\hline N. lovaniensis & Stevens, De Jonckheere y Willaert, 1980 & 45 & + \\
\hline N. australiensis & De Jonckheere, 1981 & 42 & + \\
\hline N. italica & De Jonckheere, Pernin, Scaglia y Michel, 1984 & 42 & + \\
\hline N. andersoni & De Jonckheere, 1988 & 40 & + \\
\hline N. jamiesoni & De Jonckheere, 1988 & 42 & + \\
\hline N. clarki & De Jonckheere, 1994 & 37 & + \\
\hline N. galeacystis & De Jonckheere, 1994 & 35 & + \\
\hline N. minor & De Jonckheere y Brown, 1995 & 38 & Se divide \\
\hline N. pussardi & Pernin y De Jonckheere, 1996 & 41 & + \\
\hline N. carteri & Dobson, Robinson y Rowan-Kelly,1997 & 45 & + \\
\hline N. morganensis & Dobson, Robinson y Rowan-Kelly,1997 & 44 & + \\
\hline N. niuginensis & Dobson, Robinson y Rowan-Kelly,1997 & 45 & + \\
\hline N. sturti & Dobson, Robinson y Rowan-Kelly,1997 & 44 & + \\
\hline N. robinsoni & De Jonckheere y Brown, 1999 & 38 & Se divide \\
\hline N. fultoni & De Jonckheere, Brown, Dobson, Robinson y Pernin, 2001 & 35 & + \\
\hline N. chilensis & De Jonckheere, Brown, Dobson, Robinson y Pernin, 2001 & 30 & - \\
\hline N. indonesiensis & De Jonckheere, Brown, Dobson, Robinson y Pernin, 2001 & 38 & - \\
\hline N. tihangensis & De Jonckheere, 2002 & 42 & + \\
\hline N. pringsheimi & De Jonckheere, 2002 & 37 & + \\
\hline N. pagei & De Jonckheere, 2002 & 37 & + \\
\hline N. philippinensis & De Jonckheere, 2002 & 40 & + \\
\hline N. gallica & De Jonckheere, 2004 & 33 & + \\
\hline N. americana & De Jonckheere, 2004 & 35 & + \\
\hline N. mexicana & De Jonckheere, 2004 & & + \\
\hline N. schusteri & De Jonckheere, 2004 & 37 & + \\
\hline N. dobsoni & De Jonckheere, 2004 & $>35$ & + \\
\hline N. byersi & De Jonckheere, 2004 & 42 & + \\
\hline N. endoi & De Jonckheere, 2004 & 45 & + \\
\hline N. laresi & De Jonckheere, 2004 & 42 & + \\
\hline N. martinezi & De Jonckheere, 2004 & 45 & + \\
\hline N. johanseni & De Jonckheere, 2004 & 45 & + \\
\hline N. antartica & De Jonckheere, 2004 & 28 & + \\
\hline N. dunnebackei & Visvesvara, De Jonckheere, Marciano-Cabral y Schuster, 2005 & 37 & + \\
\hline N. angularis & De Jonckheere y Brown, 2005 & 40 & + \\
\hline N. tenerifensis & De Jonckheere, 2006 & 42 & + \\
\hline N. canariensis & De Jonckheere, 2006 & 37 & + \\
\hline N. polaris & De Jonckheere, 2006 & $<30$ & + \\
\hline N. neopolaris & De Jonckheere, 2006 & $<30$ & + \\
\hline N. arctica & De Jonckheere, 2006 & $<30$ & - \\
\hline N. spitzbergenensis & De Jonckheere, 2006 & $<30$ & + \\
\hline N. neodobsoni & De Jonckheere, 2006 & $<30$ & + \\
\hline N. neoantarctica & De Jonckheere, 2006 & $<30$ & + \\
\hline N. neochilensis & De Jonckheere, 2006 & $<30$ & - \\
\hline N. paradobsoni & De Jonckheere, 2006 & $<30$ & - \\
\hline
\end{tabular}


10 en el vaso Aguacaliente y otro dique en Potrero.

Durante el proceso de secuenciación se incluyeron 2 cepas de Naegleria aisladas de la región centro del estado de Sonora, una del área de bahía Kino (Los Pingüinos), y otra del río Sonora. Se tomó una muestra de cada sitio durante el tiempo comprendido entre los meses de junio a septiembre de 2004, excepto en Las Palmas y La Isleta, sitios en los que el muestreo fue mensual. Las muestras fueron superficiales y se tomaron en la orilla, removiendo primeramente los sedimentos y dejando asentar un poco éstos, con el fin de despegar las amibas adheridas a rocas y plantas. Se utilizaron recipientes estériles plásticos de $500 \mathrm{ml}$ con tapa de rosca y se midió la temperatura del agua con un termómetro de precisión. Las muestras fueron transportadas a temperatura ambiente para su procesamiento en un tiempo no mayor de 24 horas. Cada muestra fue homogenizada por agitación vigorosa manual entre 30 y 60 segundos para asegurar el desprendimiento de las amibas de las paredes del recipiente; se pasaron $50 \mathrm{ml}$ a un tubo estéril con fondo cónico y se centrifugaron a 1000 xg durante $10 \mathrm{~min}$. Se decantó el sobrenadante dejando de 4 a $5 \mathrm{ml}$ del mismo para resuspender el sedimento. Se sembraron $0.5 \mathrm{ml}$ de la suspensión en placas de agar no nutritivo con Escherichia coli (NNE) al $2 \%$, por duplicado, a temperaturas de 28,34 y $42^{\circ} \mathrm{C}$. A las 24 y 48 horas se observaron al microscopio; se transfirieron las amibas con características del género Naegleria a nuevas placas del medio ya mencionado y se repitió esta selección hasta obtener cultivos puros. A estos aislados se les practicó la prueba de transformación ameboflagelar; los que resultaron negativos se desecharon (Page, 1988).

Para la secuenciación se seleccionaron 15 cepas representativas de los sitios de muestreo, mismas a las que se les determinó la temperatura máxima de crecimiento en cultivo monoxénico.

Para la extracción y precipitación de DNA se aplicó la técnica descrita en el manual del Dneasy Tissue Kit (Qiagen) con la modificación de usar UNSET buffer en lugar de PBS en el primer paso. El DNA se amplificó con iniciadores diseñados para detectar Naegleria spp. (De Jonckheere, 1994; De Jonckheere y Brown, 1998), además se secuenciaron usando las regiones del espaciador interno del transcrito (ITS), incluyendo la 5.8S rDNA. Los amplicones o productos de PCR fueron visualizados en gel de agarosa al $1 \%$ mediante una electroforesis del $20 \%$ de producto de PCR teñido con bromuro de etidio. El producto restante fue preparado para secuenciación usando el Qiaquick PCR clean-up kit (Qiagen, Inc., Valencia, Cal.). El producto de PCR fue secuenciado (ambas cadenas) con iniciadores de amplificación, sin clonar, en un equipo secuenciador marca Beckman CEQ2000 y usando el CEQ Dye Terminador Cycle Sequencing kit (Beckman Coulter Inc., Fullerton, Cal.). Las secuencias fueron alineadas usando el Eyeball Sequence Editor (ESEE). Las secuencias se compararon y registraron en la base de datos de secuencias nucleotídicas del laboratorio europeo de biología molecular (EMBL-NSD).

\section{Resultados}

Se obtuvieron 69 aislados con prueba de flagelación positiva, a partir de muestras de agua con temperaturas que variaron de los 29 a $45^{\circ} \mathrm{C}$ (Cuadro 2). Los muestreos

Cuadro 2. Número de ameboflagelados aislados por sitios de muestreo en Sonora, durante junio a septiembre de 2004

\begin{tabular}{lcccc}
\hline Sitio & Clave & Coordenadas & Temperatura del agua $\left({ }^{\circ}\right.$ C $)$ & Núm. de aislamientos \\
\hline Las Palmas & LP & 12 R 610917 3068120 & 30 & 8 \\
La Isleta & LIS & 12 R 608304 3070391 & 29 & 37 \\
Presa Álvaro Obregón & Pr & 12 R 609657 3078048 & 30 & 5 \\
Parque Oviachic & PO & 12 R 608897 3077585 & 30 & 0 \\
Represa Chiculi & Ch & 12 R 608493 3072579 & 29 & 0 \\
El Álamo & Al & 12 R 6084303069649 & 29 & 3 \\
Aguacaliente & AC & 12 R 614647 3068051 & 45 & 2 \\
Dique El Potrero & Di & 12 R 609797 3075435 & 32 & 5 \\
Dique 10 & Di(10) & 12 R 609419 3069306 & 33 & 4 \\
Los Pingüinos & Pin & 12 R 401148 3192595 & ND & 2 \\
Río Sonora & RS & 12 R 532822 3341109 & ND & 3 \\
Total & & & & $\mathbf{6 9}$ \\
\hline
\end{tabular}

ND: no determinado 
mensuales de los sitios de Las Palmas y La Isleta, no arrojaron datos significativos para observar algún comportamiento poblacional gradual.

Para preseleccionar a los aislados que se identificarían por secuenciación se les realizó la prueba de temperatura máxima de crecimiento, obteniéndose 43 aislados que crecieron a una temperatura máxima de $45^{\circ} \mathrm{C}$, y los 26 aislados restantes crecieron en un rango de temperatura entre 35 y $42{ }^{\circ} \mathrm{C}$. De las 3 temperaturas utilizadas para el primo aislamiento $\left(28,34\right.$ y $\left.42{ }^{\circ} \mathrm{C}\right)$, no se aisló ningún ameboflagelado a la temperatura de $28{ }^{\circ} \mathrm{C}$, debido tal vez a la gran competencia entre hongos, bacterias y otras amibas detectados a esta temperatura de incubación y a que la temperatura del agua al momento de la toma de muestra fue mayor de $28{ }^{\circ} \mathrm{C}$ en todos los sitios, lo que naturalmente eliminaría amibas que crecen por debajo de tales temperaturas.

Para la secuenciación se seleccionaron 15 cepas representativas de los sitios de muestreo con los siguientes resultados mostrados en el Cuadro 3. Además del lugar de aislamiento, se tomó como criterio de inclusión en las cepas a identificar el crecimiento a $45^{\circ} \mathrm{C}$, por ser la temperatura a la que crece $N$. fowleri, agente causal de la MAP.

\section{Discusión}

La decisión de estudiar los ameboflagelados se hizo con el fin de facilitar la búsqueda del mayor número de especies, dejando para otro estudio la búsqueda intencionada de las cepas y especies de Naegleria que no flagelan. De las cepas secuenciadas, con excepción de una sola cepa que creció a $35^{\circ} \mathrm{C}$, todas las demás se localizaron en el grupo de amibas termofílicas, lo cual concuerda con las temperaturas que predominan en la región en la época del año en que se realizaron los muestreos. Aunque es la primera ocasión en la que se registra $N$. americana (De Jonckheere) y $N$. tihangensis (De Jonckheere) en el estado de Sonora, las 2 especies parecen ser ubicuas ya que $N$. americana se ha registrado anteriormente en Europa, Australia y Norteamérica, mientras que $N$. tihangensis, en Europa, Asia, África y Norteamérica (De Jonckheere, 2004). Otras especies de Naegleria tolerantes a altas temperaturas, $N$. australiensis y $N$. lovaniensis (Stevens, De Jonckheere y Willaert), pudieron ser identificadas por secuenciación entre las aisladas previamente en México (De Jonckheere y Rivera 1984; Rivera et al., 1989). Una de las cepas de Naegleria aisladas en México, sin tipificar,

Cuadro 3. Identificación de amibas por secuenciación genética

\begin{tabular}{|c|c|c|c|c|}
\hline Сера & Origen & $\begin{array}{l}\text { Temperatura máxima de } \\
\text { crecimiento }{ }^{\circ} \mathrm{C}\end{array}$ & Especie & $\begin{array}{c}\text { Número. de correspondencia } \\
E M B L-N S D\end{array}$ \\
\hline ALIS 10013 & La Isleta & 45 & N. lovaniensis & X96568 \\
\hline BLIS 1011 & La Isleta & 45 & N. lovaniensis & X96568 \\
\hline CLIS 10052 & La Isleta & 45 & N. lovaniensis & X96568 \\
\hline DLIS 1012 & La Isleta & 45 & N. lovaniensis & X96568 \\
\hline DLIS 1013 & La Isleta & 45 & N. lovaniensis & X96568 \\
\hline DLP 10011 & Las Palmas & 45 & N. lovaniensis & X96568 \\
\hline ELIS 10042 & La Isleta & 45 & N. lovaniensis & X96568 \\
\hline $\mathrm{AC} 3$ & Aguacaliente & 45 & N. lovaniensis & X96568 \\
\hline $\operatorname{ADi}(10) 1$ & Dique 10 & 42 & N. tihangensis & AJ566631 \\
\hline $\mathrm{BAl} 2$ & El Alamo & 42 & N. tihangensis & AJ566631 \\
\hline BDi5 & Dique El Potrero & 42 & N. tihangensis & AJ566631 \\
\hline $\mathrm{BPr} 5$ & Presa Álvaro Obregón & 41 & N. tihangensis & AJ566631 \\
\hline IS2 & La Isleta & 42 & N. tihangensis & AJ566631 \\
\hline Pin 2 & Los Pingüinos & 35 & N. americana & AJ566623 \\
\hline RS49B & Río Sonora & 45 & N. lovaniensis* & X96568* \\
\hline
\end{tabular}

* Diferencia de un par de bases, una deleción de T en el pb 80 de la secuencia ITS2. 
se usó para la descripción de $N$. mexicana (De Jonckheere, 2004). La temperatura de primo aislamiento para la cepa Pin2 fue de $34^{\circ} \mathrm{C}$ y su temperatura máxima de crecimiento fue de $35^{\circ} \mathrm{C}$, lo cual concuerda con lo registrado en la bibliografía para la especie mesofílica $N$. americana, que al parecer es también la primera vez que se aísla en México. Page, en 1965 aisló por primera vez esta especie en los Estados Unidos de América, pero también se ha encontrado en Francia y Australia; sin embargo, recientemente fue nombrada como nueva especie (De Jonckheere, 2004). En México, $N$. tihangensis se aisló por primera vez en 1980 y 1983 por De Jonckheere (no publicado), quien también la había aislado en Bélgica por la misma época, en su intento de aislar $N$. fowleri a $44{ }^{\circ} \mathrm{C}$, pero no se publicaron estos hallazgos. La temperatura máxima de crecimiento de esta amiba es de $42{ }^{\circ} \mathrm{C}$, lo cual concuerda con lo determinado en este estudio. El nombre de esta especie se refiere a Tihange, Bélgica; anteriormente se le conocía como la "especie hermana" de $N$. australiensis, con quien está cercanamente emparentada.

Naegleria lovaniensis había sido identificada en nuestra región por Lares-Villa et al. (1997). Es la especie más cercanamente emparentada con $N$. fowleri y se encuentra globalmente distribuida con muy poca variación en su secuencia de rDNA intraespecie (De Jonckheere, 2002). Por lo tanto, es importante mencionar que se registra por primera vez el aislamiento de una cepa de $N$. lovaniensis del río Sonora, con una diferencia de un par de bases en la secuencia ITS2 (una deleción de T en una repetición de tres T), comparada a todas las cepas aisladas previamente.

Otras especies ya aisladas en el estado de Sonora, incluyen $N$. fowleri, $N$. gruberi y $N$. australiensis (LaresVilla et al., 1997; Vargas-Zepeda, et al., 2005); lo cual nos da un total de 6 especies conocidas hasta el momento. A pesar de que en este estudio no se pudo identificar $N$. fowleri, debido a la gran cantidad de amibas termofílicas, especialmente $N$. lovaniensis, que se puede tomar como un indicador de la presencia de $N$. fowleri, no se puede despreciar el riesgo de nadar en aguas que ofrecen las condiciones necesarias para su crecimiento.

Se recomienda para estudios posteriores tomar las muestras en épocas del año con temperaturas menores a las del verano, para poder aislar amibas mesofílicas que pudiesen estar en menor concentración, y así conocer mejor la diversidad del género Naegleria.

\section{Literatura citada}

De Jonckheere, J. F. 1994. Riboprinting of Naegleria spp.: small subunit versus large subunit. Parasitology Research 80:230234.

De Jonckheere, J. F. 2002. A century of research on the amoeboflagellate genus Naegleria. Acta Protozoologica 41:309-342.

De Jonckheere, J. F. 2004. Molecular definition and the ubiquity of species in the genus Naegleria. Protist 155:89-103.

De Jonckheere, J. F. 2006a. Isolation and molecular identification of vahlkampfiid amoebae from an island (Tenerife, Spain). Acta Protozoologica 45:91-96.

De Jonckheere, J. F. 2006b. Isolation and molecular identification of free-living amoeba of the genus Naegleria from arctic and sub-antarctic regions. European Journal of Protistology 42:115-123.

De Jonckheere, J. F. y F. Rivera. 1984. Thermophilic Naegleria in a recreation center in the vicinity of Mexico city. Journal of Protozoology 31:68A-69A (Abstract 248).

De Jonckheere, J. F. y S. Brown. 1998. Three different group I introns in the nuclear large subunit ribosomal DNA of the amoeboflagellate Naegleria. Nucleic Acids Research 26:456-461.

De Jonckheere, J. F. y S. Brown. 2005. Description of a new species with a remarkable cyst structure in the genus Naegleria: Naegleria angularis sp. n. Acta Protozoologica 44:61-65.

De Jonckheere, J. F., S. Brown, P. J. Dobson, B. S. Robinson y P. Pernin. 2001. The amoeba-to flagellate transformation test is not reliable for the diagnosis of the genus Naegleria. Description of three new Naegleria spp. Protist 152:115-121.

Lares-Villa, F., A. M. Guerrero-Dumás y A. López-Osorio. 1997. Estudio protozoológico (subphylum Sarcodina clase Lobosea), en ambientes acuáticos de la región del Valle del Yaqui, Sonora, México. Instituto Tecnológico de SonoraDirección de Investigación y Estudios de Posgrado 2:47-55.

Page, F. C. 1988. A new key to freshwater and soil gymnamoebae. CCAP, Ambleside, Cumbria. 122 p.

Rivera, F., F. Lares, E. Gallegos, E. Ramírez, P. Bonilla, A. Calderón, J. J. Martínez, S. Rodríguez y J. Alcocer. 1989. Pathogenic amoebae in natural thermal waters of three resorts of Hidalgo, Mexico. Environmental Research 50:289-295.

Vargas-Zepeda, J., A. Gómez-Alcalá, J. A. Vázquez-Morales, L. Licea-Amaya, J. F. De Jonckheere y F. LaresVilla. 2005. Successful treatment of Naegleria fowleri meningoencephalitis by using intravenous amphotericin B, fluconazole and ifampicin. Case report. Archives of Medical Research 36:83-86.

Visvesvara, G. S., J. F. De Jonckheere, R. Sriram y B. Daft. 2005. Isolation and molecular typing of Naegleria fowleri from the brain of a cow that died of primary amebic meningoencephalitis. Journal of Clinical Microbiology 43:203-4204. 